\title{
Quantitative microdialysis determination of extracellular striatal dopamine concentration in male and female rats: effects of estrous cycle and gonadectomy
}

\author{
Li Xiao ${ }^{\mathrm{a}, \mathrm{b}}$, Jill B. Becker ${ }^{\mathrm{a}-\mathrm{c}, *}$ \\ ${ }^{a}$ Psychology Department, ${ }^{b}$ Reproductive Sciences Program and Neuroscience Program, University of Michigan, Ann Arbor, MI, USA
}

Received 29 June 1994; Revised version received 25 August 1994; Accepted 25 August 1994

\begin{abstract}
Sex differences in basal extracellular striatal dopamine concentrations in gonadectomized male and female rats have been reported previously. In the current experiment, estrous cycle-dependent variation, sex differences and the effect of gonadectomy on extracellular striatal dopamine concentrations were determined using quantitative microdialysis. Female rats were found to have significantly higher extracellular striatal dopamine concentrations in proestrus and estrus than in diestrus or after ovariectomy. In contrast, castration of male rats had no effect on extracellular striatal dopamine concentrations. Thus, endogenous ovarian hormones, but not testicular hormones, modulate extracellular striatal dopamine concentrations in rats.
\end{abstract}

Key words: Estrous cycle; Ovariectomy; Castration; Ovarian hormone; Extracellular striatal dopamine; Quantitative microdialysis

Ovarian hormones have been shown to modulate behavioral and neurochemical indices of activity in the nigrostriatal dopamine (DA) system. For example, DA agonist-stimulated behaviors, such as rotational behavior, general activity and stereotypy, vary with the estrous cycle $[1-7,13,18]$. Female rats in estrus exhibit less general activity and greater intensity of stereotyped behavior after amphetamine (AMPH) treatment than do animals in diestrus [4]. Estrous females also show a greater number of AMPH-induced rotations than do animals $24 \mathrm{~h}$ later on the 1st day of diestrus [7]. Ovariectomy (OVX) attenuates rotational behavior induced by AMPH, compared with the response of estrous females, and estrogen treatment in OVX rats potentiates AMPH-induced rotational behavior $[1,2]$.

The hormonal modulation of AMPH-induced behaviors in female rats is correlated with changes in striatal DA release. AMPH-induced DA release from striatal tissue in vitro varies with the phase of the estrous cycle

*Corresponding author. Address: Neuroscience Laboratory Building, 1103 East Huron Street, Ann Arbor, MI 48104-1687, USA.

Fax: (1) (313) 936-2690. E-mail: JBBecker@umich.edu
[4,5], OVX attenuates AMPH-induced DA release [5] and estrogen treatment enhances AMPH-induced DA release from striatal tissue in vitro [2]. Studies using in vivo microdialysis also find that $\mathrm{AMPH}$-stimulated DA release in striatum is enhanced on estrus compared with other days of the female rat's estrous cycle [4] and a single estrogen treatment enhances striatal DA release induced by AMPH $30 \mathrm{~min}$ later $[1,10]$. By contrast, castration (CAST) of male rats either has no effect or enhances striatal DA-mediated behaviors $[9,11]$ and AMPH-induced striatal DA release [5].

In a previous study, when CAST and OVX rats were compared, there was a sex difference in basal DA concentrations in striatum determined by in vivo quantitative microdialysis [10]. CAST males had significantly higher basal striatal DA than OVX females. The current experiment examines whether there are sex differences in the extracellular striatal DA concentrations in intact male and female rats and whether there is estrous cycledependent variation in extracellular striatal DA concentrations in female rats. To answer these questions, the present experiment applied the quantitative in vivo microdialysis technique (the 'no net flux' method $[14,15]$ ) to examine extracellular striatal DA concentrations in 
the striatum of freely moving CAST and intact male rats as well as OVX and intact female rats at each stage of the estrous cycle. The no net flux procedure applied here provides a more accurate estimation of the extracellular DA concentrations than standard microdialysis procedures [14.15]

Adult male and female Holtzman rats $(150200 \mathrm{~g}$ : Sprague-Dawley, Indianapolis, IN) were maintained on a 14:10 light:dark cycle (lights on at 06:00) with food and water ad libitum. Vaginal smears were taken daily from intact female rats to monitor the stage of estrous cycle. Female rats with regular 4-day estrous cycles were used on estrus (day of behavioral receptivity), diestrus (either of the 2 days of the follicular phase that immediately follows estrus) and proestrus (the day preceeding estrus). All other animals were handled daily at the same time. All procedures were carried out according to an approved 'use and care of animals' protocol.

Female rats assigned to the OVX group and male rats assigned to the CAST group were gonadectomized under light ether anesthesia supplemented with methoxyflurane and were allowed at least 2 weeks recovery before to further manipulation. Vaginal smears were taken from OVX females for at least 10 consecutive days and any animals showing cornified cells in the vaginal smear were excluded from the study. Guide cannulae were chronically implanted bilaterally, aimed at the dorsolateral striatum, as described previously [10]. Animals were allowed at least 4 weeks recovery before microdialysis and daily vaginal smears or handling were continued during this period of time. A modified design [12] of a removable. concentric-style microdialysis probe [17] was used in this experiment. In vitro recovery was conducted and only probes with recovery of $10-20 \%$ were used for microdialysis in vivo, to minimize the variation among probes.

On the afternoon before microdialysis, animals were transferred to a room maintained under the same conditions of temperature and light:dark cycle as in the animal colony. Dialysis probes were then inserted bilaterally into the striatum via the guide cannulae. A Ringer's solution (145 mM NaCl, $2.7 \mathrm{mM} \mathrm{KCl}, 1.2 \mathrm{mM} \mathrm{CaCl}$. $1.0 \mathrm{mM} \mathrm{MgCl}{ }_{2}, 0.25 \mathrm{mM}$ ascorbic acid; $\mathrm{pH}=7.3$ ) was delivered at a rate of $0.2 \mu \mathrm{l} / \mathrm{min}$ by a microinfusion pump overnight. Freely moving rats were kept individually in dialysis chambers with food and water available throughout the entire microdialysis procedure. Animals from 2-3 of the groups (except for estrus females and intact males that were not tested together) were run simultaneously on a given day. At least three vaginal smears were taken from each intact female rat (at 08:00, 12:00 and 18:00) on the day of dialysis to reconfirm the stage of the estrous cycle. $\sim 16 \mathrm{~h}$ after probe insertion (i.e.. at 08:00 on the next day), the microinfusion pump was turned up to $1.5 \mu \mathrm{l} / \mathrm{min}$. After a stable basal DA level was reached, the perfusate was changed in a random sequence 10 a Ringer's solution containing either 4 or 16

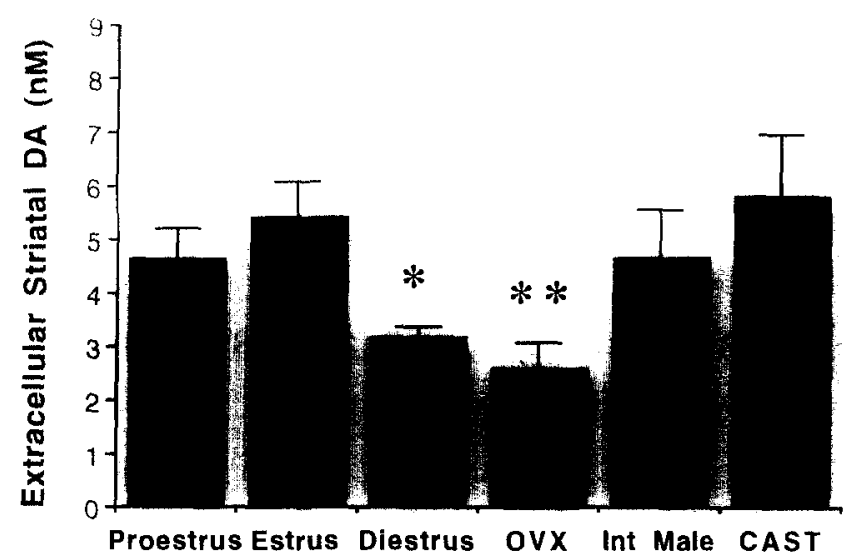

Fig. 1. There is an estrous cycle-dependent fluctuation in extracellular striatal DA concentrations (nM: $\left.F_{2,24}=6.38 . P<0.01\right)$. *Females in diestrus $(3.17 \pm 0.20$; mean \pm S.E.M.; $n=11)$ showed significantly lower extracellular DA than those in estrus $(5.42 \pm 0.67 ; n=9$ : $P<0.002)$ and those in proestrus $(4.63 \pm 0.56 ; n=7 ; \quad P<0.05)$ * OVX resulted in a significant reduction in extracellular DA $(2.59 \pm 10.50, n=7)$ when compared with females in estrus $(P<0.001)$ or proestrus $(P<0.05)$ while there was no difference between intact (Int Male: $4.67 \pm 0.55 ; n=8)$ and CAST males $(5.82 \pm 1.16 ; n=10$ : $P=0.40$ ) in extracellular DA concentrations in dorsolateral striatum. Extracellular DA was significantly higher in CAST males than in OVX $(P<0.02)$ or diestrus females $(P<0.02)$. Bars indicate mean \pm S.E.M.

nM DA. A 90-min equilibrium period (determined by our pilot data) was required after switching the perfusate and then three 30-min samples were collected for each DA concentration in the perfusate. All samples were injected by an autosampler (ESA, Chelmsford, MA) onto a high-performance liquid chromatography system with coulometric detection (HPLC-EC; ESA) to analyse DA content. Solutions were changed when the variation in DA concentration found in three consecutive dialysate samples was $<10 \%$.

Brains were formalin ( $10 \%$ ) perfused and $40-\mu \mathrm{m}$ sections were stained with Cresyl violet. Data from probes that were not located entirely within the desired brain region or animals with damage in the brain area around the probe on either side of the striatum were eliminated from the study.

Concentrations of DA in dialysate samples were plotted as a function of the DA perfused through the probe. The point corresponding to an individual animal's extracellular DA concentration was the concentration at which the net flux of DA across the dialysis probe membrane was zero; this was calculated using simple linear regression [10]. A one-way ANOVA and posthoc pairwise comparisons (Fisher's test) were used for comparing the differences in extracellular DA concentrations among the groups.

As indicated in Fig. 1, there is estrous cycle-dependent fluctuation in extracellular striatal DA concentrations $(P<0.01)$. Females in estrus showed significantly higher 
extracellular DA than those in diestrus $(P<0.002)$. Females in proestrus also had higher extracellular DA than diestrous females $(P<0.05)$ while no significant difference in DA concentrations in striatum was observed between females in proestrus and estrus. OVX resulted in a significant reduction in extracellular DA when compared with females in estrus $(P<0.001)$ or proestrus $(P<0.05)$ while there was no difference between intact and CAST males in extracellular DA concentrations in dorsolateral striatum (see legend of Fig. 1 for additional statistical details).

Sex differences in extracellular striatal DA were found between CAST and OVX groups $(P<0.02)$ as well as between CAST males and diestrus females $(P<0.02)$. There were no differences between the intact males and the female groups, due to the large variation in the extracellular striatal DA concentrations in the male group. Interestingly, if the basal extracellular DA concentrations for the intact males $(n=12 ; 4.67 \pm 0.92 \mathrm{nM})$ and all of the intact females $(n=27 ; 4.30 \pm 0.33 \mathrm{nM})$ are compared, they do not differ $(P=0.639)$. Note also that females do not exhibit greater variability than males even when data from all days of the estrous cycle are included.

The results of this experiment demonstrate for the first time estrous cycle-dependent variation in the extracellular DA concentration, using quantitative microdialysis in vivo. Female rats in both proestrus and estrus had significantly higher extracellular DA in dorsolateral striatum than those in diestrus. Depletion of the endogenous source of ovarian hormones by OVX was associated with an attenuation of extracellular DA compared with intact females in proestrus or estrus. By contrast, intact male rats and CAST males did not differ in their extracellular DA concentration in dorsolateral striatum. These results support the notion that ovarian hormones, but not testicular hormones, modulate DA activity in the nigrostriatal system and complement results from previous studies that have found estrous cycle-dependent variation in striatal DA release and DA-mediated behaviors.

Sex differences and the effect of estrous cycle on extracellular DA in the striatum may reflect changes in DA release, metabolism, reuptake or diffusion properties within the striatum. Evidence from Castner et al. [10] indicates that DA reuptake does not contribute to sex differences in basal extracellular DA in OVX and CAST, but it is not known whether the effect of estrous cycle is due to variation in DA reuptake. Regardless of the mechanism, the available data indicate that ovarian hormones have both rapid and prolonged effects on striatal DA activity. In the current study, basal DA concentrations were found to be higher both on the afternoon of proestrus when plasma estradiol and progesterone are known to reach their maximum and $24 \mathrm{~h}$ later on estrus when they are relatively low. Previous research from this laboratory has found that AMPH-stimulated DA release from striatal tissue is lower on the morning of proestrus than on the night of behavioral estrus [5], suggesting changes in striatal DA activity during the estrous cycle are concomitant with the proestrous afternoon increases in estrogen and progesterone.

Enhanced behavioral responsiveness has been reported on proestrus and estrus when there is enhanced extracellular striatal DA. For example, electrical stimulation of the nigrostriatal bundle induces DA-dependent rotational behavior and this turning behavior is greater during estrus and proestrus than during diestrus [16]. AMPH-induced turning behavior is also enhanced on estrus and proestrus relative to diestrus [7]. Finally, sensorimotor performance, as assessed by footfaults made while traversing a suspended narrow beam, is superior on the evening of estrus compared with diestrus; performance on proestrus was in between that of estrus and diestrus [8]. Thus, during the estrous cycle, enhanced sensorimotor performance and enhanced behavioral responsiveness to stimulation apparently occur during those periods when extracellular striatal DA is greatest.

This research was supported by NSF Grant BNS 9021966 (to J.B. Becker).

[1] Becker, J.B., Estrogen rapidly potentiates amphetamine-induced striatal dopamine release and rotational behavior during microdialysis, Neurosci. Lett., 118 (1990) 169-71.

[2] Becker, J.B. and Beer, M.E., The influence of estrogen on nigrostriatal dopamine activity: behavioral and neurochemical evidence for both pre- and postsynaptic components. Behav. Brain Res., 19 (1986) 27-33

[3] Becker, J.B., Beer, M.E. and Robinson, T.E., Striatal dopamine release stimulated by amphetamine or potassium: influence of ovarian hormones and the light-dark cycle, Brain Res., 311 (1984) $157-160$

[4] Becker, J.B. and Cha, J., Estrous cycle-dependent variation in amphetamine-induced behaviors and striatal dopamine release assessed with microdialysis. Behav. Brain Res.. 35 (1989) 117 125.

[5] Becker, J.B. and Ramirez, V.D.. Sex differences in the amphetamine stimulated release of catecholamines from rat striatal tissue in vitro, Brain Res., 204 (1980) 361-72.

[6] Becker, J.B. and Ramirez, V.D.. Experimental studies on the development of sex differences in the release of dopamine from striatal tissue fragments in vitro. Neuroendocrinology, 32 (1981) 168 173.

[7] Becker, J.B., Robinson, T.E. and Lorenz, K.A., Sex differences and estrous cycle variations in amphetamine-elicited rotational behavior, Eur. J. Pharmacol., 80 (1982) 65 72.

[8] Becker. J.B., Snyder, P.J., Miller. M.M., Westgate, S.A. and Jenuwine, M.J., The influence of estrous cycle and intrastriatal estradiol on sensorimotor performance in the female rat. Pharmacol. Biochem. Behav.. 27 (1987) 53-59.

[9] Camp, D.M., Becker, J.B. and Robinson. T.E.. Sex differences in the effects of gonadectomy on amphetamine-induced rotational behavior in rats, Behav. Neural Biol., 46 (1986) $491-495$.

[10] Castner, S.A., Xiao, L. and Becker, J.B., Sex differences in striatal dopamine: in vivo microdialysis and behavioral studies. Brain Res., 610 (1993) 127-134.

[11] Dluzen, D.E. and Ramirez, V.D.. Effects of orchidectomy on nigrostriatal dopmainergic function: behavioral and physiological evidence, J. Neuroendocrinol., 1 (1989) 285-290 
[12] Meisel, R.L., Camp. D.M. and Robinson, T.E.. A micordialysic study of ventral striatal dopamine during sexual behaivor in female Syrian hamsters, Behav. Brain Res., 55 (1993) 151157.

[1.3] Miller. J.C.. Sex differences in dopaminergic and cholinergic activity and function in the nigrostriatal system of the rat. Psychneuroendocrinology, 8 (1983) 225236

[14] Morrison, P.F. Bungay, P.M.. Hsiao, I.K.. Mefford. I.N. and Dystra, K.H. Quantitative microdialysis, In T.E. Robinson and J.B. Justice (Eds.). Microdialysis in the Neurosciences. Elsevier, Amsterdam, The Netherlands, 1991, pp. 211214.

[15] Parsons, L.H. and Justice. J. B.. Quantitative neurotransmitter microdialysis: extracellular dopamine in the nucleus accumbens, J. Neurochem., 58 (1992) 212218.
[16] Robinson, T.E., Camp, D.M., Jacknow. D.S. and Becker, J.B., Sex differences and estrous cycle dependent variation in rotational behavior elicited by electrical stimulation of the mesostriatal dopamine system, Behas. Brain Res. 6 (1982) 273 287.

[17] Robinson. T.E. and Whishaw. I.Q., Normalization of extracellular dopamine in striatum following recovery from a partial unilateral 6-OHDA lesion of the substantia nigra: a microdialysis study in freely moving rats. Brain Res., 450 (1988) 209 224.

[18] Van Hartesveldt, C. and Joyce, J.N., Effects of estrogen on the basal ganglia, Neurosci. Biobehav. Rev., $10(1986) 114$. 